\title{
Variations
}

Variations

Revue internationale de théorie critique

$8 \mid 2006$

Subjectivités libres et critique de la répression

\section{Antoine Artous, Le fétichisme chez Marx, le marxisme comme théorie critique}

Guillaume Collinet

\section{(2) OpenEdition}

1 Journals

Édition électronique

URL : http://journals.openedition.org/variations/525

DOI : $10.4000 /$ variations. 525

ISSN : 1968-3960

Éditeur

Les amis de Variations

Édition imprimée

Date de publication : 1 novembre 2006

Pagination : 119-124

Référence électronique

Guillaume Collinet, "Antoine Artous, Le fétichisme chez Marx, le marxisme comme théorie critique », Variations [En ligne], 8 | 2006, mis en ligne le 27 décembre 2012, consulté le 22 septembre 2020. URL http://journals.openedition.org/variations/525; DOI : https://doi.org/10.4000/variations.525

Ce document a été généré automatiquement le 22 septembre 2020.

Les ami•e•s de Variations 


\title{
Antoine Artous, Le fétichisme chez Marx, le marxisme comme théorie critique
}

\author{
Guillaume Collinet
}

\section{RÉFÉRENCE}

Antoine Artous, Le fétichisme chez Marx, le marxisme comme théorie critique. Syllepse, Paris, 2006

Contrairement à ce que son titre pourrait laisser suggérer, l'ouvrage d'Artous ne se limite pas à un simple travail d'exégèse sur l'un des concepts les plus fameux du corpus marxien, celui de fétichisme. Son objectif est plutôt d'éprouver la pertinence de ce concept pour penser les rapports sociaux capitalistes. En discutant certains prolongements donnés à la théorie marxienne du fétichisme, par Lukács et Pakusanis notamment, et en montrant que cette théorie permet d'engager un dialogue fécond avec des auteurs non-marxistes, comme Weber ou Foucault, Artous souligne la richesse de cette catégorie de fétichisme, y compris pour penser un certain nombre de points aveugles légués par la tradition marxiste.

\section{Retour sur la théorie marxienne de la valeur}

D'abord, pourquoi s'intéresser spécifiquement à la catégorie de fétichisme ? C'est que, pour Artous, cette catégorie se trouve au centre de la problématique de Marx. Il prend à rebours aussi bien les lectures orthodoxes que celles d'inspiration althussérienne, qui tendent à interpréter le passage du Capital consacré au fétichisme comme une digression d'ordre philosophique (plus ou moins heureuse), dans un ouvrage à vocation scientifique. En fait, cette question du statut de la théorie du fétichisme, au sein de l'ensemble de la conceptualisation marxienne, est liée à celle, plus fondamentale, de 
l'interprétation qu'il convient de faire de la théorie marxienne de la valeur. Trop souvent, aux yeux d'Artous, cette théorie de la valeur a été lue comme une simple reprise de la théorie de la valeur-travail, déjà formulée par les classiques (Ricardo notamment). Cette lecture conduit à masquer ce qui constitue le véritable apport de Marx. Car la question qu'il pose est tout autre que celle posée par les classiques. Son problème n'est pas celui de la mesure de la valeur (ce qui revient à admettre d'emblée que les marchandises ont une valeur) ; c'est celui de l'origine, ou plutôt du fondement de la valeur. Pourquoi, dans les sociétés capitalistes, les produits du travail prennent-ils la forme de marchandises, sont objectivés socialement comme dotés d'une valeur? Réponse de Marx : la valeur, qui paraît une qualité naturelle, inhérente aux produits du travail, n'est en fait que la représentation objectivée socialement du travail abstrait, c'est-à-dire de la forme que prend le travail social en régime capitaliste. En d'autres termes, la valeur cristallise les rapports sociaux qui se nouent dans le procès de travail capitaliste. Marx opère donc, au travers de sa théorie de la valeur, une dénaturalisation des rapports marchands, ce qui contraste bien sûr avec la préoccupation qui était celle des classiques. Artous qualifie la théorie marxienne de "théorie de la forme valeur des produits du travail » à la suite de Jean-Marie Vincent, à qui il dédie son livre.

\section{Désenchantement du monde et fétichisme}

3 On comprend mieux, dès lors, pourquoi le fétichisme occupe une place cardinale. $\mathrm{Ce}$ concept ne désigne rien d'autre, en effet, que le voile pudique jeté sur cette origine de la valeur. Plus précisément, ce concept a vocation à rendre compte de la forme d'opacité spécifique qui caractérise les rapports sociaux capitalistes. Chaque système social engendre des formes d'opacités spécifiques. En ce qui concerne les formes précapitalistes, cette opacité tient à l'inscription des rapports sociaux de production dans des rapports de dépendance personnelle (maître/esclave, seigneur/serf...), euxmêmes légitimés en référence à un ordre naturel ou cosmique. Force est de constater que, de ce point de vue, le capitalisme représente une rupture. L'ère bourgeoise est marquée par un mouvement de "désenchantement du monde " (Weber). Marx enregistre parfaitement cette rupture; c'est pourquoi il souligne que le salarié moderne ne vend pas sa personne, mais sa seule force de travail (le rapport salarial n'est donc pas assimilable à une dépendance personnelle). Pour autant, Marx voit aussi que, malgré le retrait des Dieux hors de la vie de la Cité, les rapports sociaux capitalistes ne se donnent pas pour ce qu'ils sont - à savoir des rapports d'exploitation. Au contraire, ils s'objectivent dans la représentation sociale de l'objet - et de la force de travail - comme marchandise; ils prennent la forme d'un rapport entre choses, auquel on attribue, fantasmatiquement, le pouvoir de configurer l'ordre social. C'est pour rendre compte de cette forme d'opacité spécifique, consistant à chosifier un rapport social, que Marx mobilise la catégorie de fétichisme, initialement destinée à caractériser les formes de religiosité les plus primitives et le fait d'adorer un objet auquel on attribue des pouvoirs divins. Cet emprunt a d'abord une fonction critique : une catégorie désignant une forme archaïque est utilisée pour appréhender certains caractères des sociétés dites les plus développées.

4 Ces formes d'opacités spécifiques générées par chaque système social, comme y insiste à juste titre Artous, ne relèvent pas d'une simple illusion, justifiant a posteriori des rapports de production qui pourraient subsister idéalement en dehors d'elles. Ces 
représentations sociales constituent les rapports sociaux qu'elles servent à légitimer. Elles en sont le présupposé, la condition. Leur réduction à un simple reflet, plus ou moins déformé, des conditions matérielles de la production, peut s'autoriser, il est vrai, de certains textes de Marx. Elle est surtout devenue un véritable lieu commun du marxisme ultérieur. Ce n'est pas l'un des moindres mérites de l'ouvrage d'Artous que de rompre définitivement avec ce lieu commun. Il établit d'ailleurs, de façon convaincante, que l'effort constant de Marx pour penser la dimension idéelle des rapports sociaux cadre mal avec cette théorie de la représentation-reflet.

\section{Fétichisme de la marchandise et fétichisme de la production}

Un des autres apports majeurs de la réflexion d'Artous concerne la théorie du fétichisme elle-même. On réduit souvent cette théorie à celle du fétichisme de la marchandise. Or, il est possible de montrer que, dans les textes qu'il consacre à la « subsomption réelle du travailleur sous le capital », Marx dévoile un second fétichisme : celui qui est propre à l'organisation capitaliste de la production. Le concept de «subsomption réelle", en effet, désigne le fait que, dans le procès capitaliste de production, le travailleur, en plus de ne pas être propriétaire des moyens de production («subsomption formelle»), perd toute maîtrise de ce procès. En d'autres termes, le travailleur se voit transformé en simple auxiliaire des moyens de production (via le machinisme, son confinement à des tâches de simple exécution, etc.). Par conséquent, ces moyens de production apparaissent comme étant eux-mêmes sources de valeur (comme en témoigne la distinction, propre à l'économie bourgeoise, du capital circulant et du capital fixe, qui cache la différence radicale entre force de travail et moyens de production). Marx suggère lui-même la parenté de cette représentation avec celle du fétichisme de la marchandise. Étant précisé que, dans ce dernier cas, le fétichisme prend la forme d'une "chosification des rapports sociaux", alors que, dans celui de l'organisation, sa forme est plutôt celle d'une «personnification des choses » (les moyens de production en tant que personnification du capital).

En dégageant un fétichisme propre à l'organisation capitaliste de la production, distinct de celui de la marchandise, Artous exploite à fond une intuition de Marx, à laquelle ce dernier, on le verra, n'a pas toujours su rester fidèle. Car cet élargissement de la théorie du fétichisme est lourd d'implications théoriques. En particulier, il conduit à rompre avec la définition marxiste classique du capitalisme comme simple généralisation des rapports marchands. S'il est vrai que l'organisation capitaliste engendre des formes d'opacité et de domination irréductibles à celles générées par les rapports marchands, il faut en conclure que le capitalisme se définit aussi par l'institution de formes organisationnelles spécifiques. Cela implique en outre que la forme organisée de production ne peut suffire à elle seule à définir le socialisme. La tradition marxiste, y compris Marx lui-même, a eu tort d'identifier celui-ci au passage de la forme marchande de production à son organisation planifiée. L'organisation elle-même peut devenir une forme sociale fétichisée, peut donner lieu à des rapports de classe. L'abolition du marché n'est pas synonyme de la fin de l'exploitation. On le voit, en soulevant l'idée d'un fétichisme de l'organisation, la réflexion d'Artous nous confronte à des questions majeures pour l'élaboration d'un « socialisme du XXIe siècle ». 


\section{Théorie du fétichisme et théorie de la réification}

$7 \quad$ Les textes de Marx consacrés à l'organisation capitaliste ont été lus attentivement par de nombreux auteurs marxistes, en particulier Lukács, qui constate et anticipe le fait que le développement du capitalisme aboutit à une minoration des rapports directement marchands, et à une augmentation de la forme organisée de production. Dans Histoire et conscience de classe, Lukács élabore une théorie de la réification destinée à tenir compte de cette évolution; il introduit à ce titre une série d'inflexions notables dans la théorie marxienne du fétichisme.

8 À la différence de Marx, Lukács part du procès de production immédiat (il élude le moment spécifique des rapports marchands). Et il repère, dans ce procès, une mise en oeuvre effrénée du « principe de rationalisation sur la base du calcul » (Weber). En d'autres termes, l'organisation capitaliste n'est pas seulement le lieu d'une extorsion du surtravail ; elle se caractérise aussi et surtout par une perte des propriétés qualitatives et individuelles du travail humain, sous l'effet de la quantification du travail opérée dans le procès de production capitaliste. Par conséquent, il faut radicaliser la théorie du fétichisme. Ce ne sont plus seulement les rapports sociaux qui se trouvent chosifiés, mais les individus eux-mêmes. Cette logique de réification ne touche pas que le procès de production. Elle tend à envahir la totalité de l'espace social, jusqu'à impliquer les formes juridiques, politiques, voire scientifiques qui accompagnent l'essor de la société bourgeoise.

9 Mais, pour Artous, cette théorie de la réification, aussi impressionnante soit-elle, sousestime les contradictions propres aux rapports sociaux capitalistes. Si, dans le rapport salarial, le travailleur est réduit à l'état de simple chose, il n'existe plus aucune différence fondamentale entre ce rapport et celui qui unit un maître et son esclave, ou un seigneur et un serf (dans lequel, effectivement, le travailleur n'est plus une personne, mais une chose). Lukács abolit la spécificité du rapport salarial d'exploitation. Le salarié moderne met à la disposition du capitaliste non sa personne tout entière, mais seulement sa force de travail. Son statut est donc contradictoire : il est à la fois sujet de droit et objet du despotisme d'usine. De même, l'analyse Lukácsienne des formes politiques de la société bourgeoise reste unilatérale. Dans le sillage de Weber, l'auteur d'Histoire et conscience de classe définit l'État moderne comme un appareil bureaucratique, dans lequel la volonté collective se trouve réifiée. Cette définition comporte assurément une part de vérité. Mais l'État moderne est aussi un État censément représentatif, fondé sur la prétention de liberté et d'égalité.

Cette critique de Lukács permet de réaffirmer une nouvelle fois que les formes de représentations sociales des rapports sociaux, tout en étant sources d'opacité, ne sont pas réductibles à de simples illusions, à des faux-semblants. Le propre de la théorie du fétichisme de Marx, c'est justement de montrer que les rapports sociaux capitalistes ne sont pas transparents à eux-mêmes, sans pour autant rabattre ces rapports sur des rapports de dépendance personnelle. L'exploitation, en changeant de visage, modifie sa nature. Dans le capitalisme, les individus paraissent dominés par des choses, des " abstractions » (Jean-Marie Vincent), mais pas par des personnes. Ce que dit précisément le concept de fétichisme. 


\section{Le sujet juridico-politique et le travailleur parcellaire}

11 On peut maintenant en venir à ce qu'Artous désigne comme "l'autre face » de la théorie du fétichisme, à savoir les formes d'individuation portées par le double mouvement de "chosification des rapports sociaux" (fétichisme de la marchandise) et de " personnification des choses" (fétichisme de l'organisation). Le procès d'échange est à l'origine d'une première forme d'individuation (comme l'indique Marx en ouverture du chapitre II de la section I du livre I du Capital). Car, dans l'échange marchand, les individus se reconnaissent réciproquement comme des individus libres et égaux. La figure du sujet juridico-politique moderne s'inscrit donc dans la relation marchande comme son présupposé. Encore une fois, il ne s'agit pas d'une simple illusion, mais d'une forme sociale objective, comme en témoigne l'existence d'institutions juridiques spécifiques (qui signale l'autonomisation de la sphère du droit dans les sociétés capitalistes). Le fétichisme de la marchandise se double d'un "fétichisme juridique " (Pakusanis), par lequel les rapports sociaux capitalistes s'annoncent comme le résultat de rapports interindividuels.

Une seconde forme d'individuation, contradictoire avec la première, naît de la « subsomption réelle du travailleur sous le capital». Au sein de l'entreprise capitaliste, le travailleur n'est plus seulement ni essentiellement un sujet juridique; il devient un objet, parmi d'autres, du despotisme d'usine. Il prend la forme du travailleur parcellaire, de l'individu standardisé. Le mécanisme social, qui sous-tend ce second procès d'individuation, se déroule dans et surtout hors de la sphère de la production : au travers de l'habitat, de l'éducation, de la santé... L'État joue un rôle clé lors de ce second procès : il impulse des politiques dans les domaines précités, et reproduit en son sein des facteurs de standardisation. Toutefois, cette production du travailleur parcellaire n'implique pas des formes de domination directe des individus. Artous mobilise, pour illustrer ce point, les analyses de Foucault. L'auteur de Surveiller et punir montre, en effet, que l'État moderne recourt à des dispositifs disciplinaires en rupture avec la domination directe sur les corps exercée par le pouvoir politique au Moyen Âge. Pour Artous, la dynamique conflictuelle du capitalisme s'inscrit dans la contradiction entre sujet juridico-politique et travailleur parcellaire. Ce qui peut être discuté. Car la figure $\mathrm{du}$ sujet juridico-politique moderne, contrairement à ce que paraît supposer Artous, n'est pas homogène. Elle n'englobe pas seulement la position de l'autonomie de la personne (liberté de vivre à sa guise, avec d'autres et en contractant avec d'autres), mais aussi celle du citoyen souverain (liberté de participer, à égalité avec les autres, à la formation de la volonté commune). Les auteurs libéraux comme Constant s'emploient à nier le caractère moderne de l'autonomie du citoyen-souverain, en la subsumant sous la catégorie de "liberté des Anciens». Erreur qui manifeste l'extériorité de la pensée libérale par rapport à l'aspiration démocratique, considérée dans sa complexité antinomique (émancipation individuelle - de l'homme - et collective - du citoyen). Erreur que reproduit, à des fins critiques Artous, en identifiant purement et simplement la figure du sujet moderne à l'individualisme marchand.

\section{Le fétichisme du plan}

Plus intéressant est l'ultime chapitre de l'ouvrage d'Artous, qui examine, à propos de la délicate question de la nature $d u$ socialisme, les implications du nécessaire 
élargissement de la théorie du fétichisme au-delà du seul fétichisme de la marchandise. À quoi ressemblerait une société débarrassée de tout fétichisme? On sait que Marx répond à cette question par l'idée de «plan concerté». Dans une société où, sur la base d'une propriété commune des moyens de production, l'activité économique prendrait une forme planifiée, les rapports sociaux deviendraient simples et transparents. En répondant ainsi, Marx se montre curieusement infidèle à son intuition d'un fétichisme propre à la forme organisée. En témoigne, notamment, la comparaison qu'il instaure entre la démarche de Robinson Crusoë, notant sur un cahier les tâches déjà effectuées et celles demeurant à accomplir, et la logique prévalant dans la future société communiste. La planification, même concertée, à l'instar de celle réalisée au sein de la firme capitaliste, correspond à une forme sociale, cristallisant le travail social obtenu par la coopération entre les divers producteurs. En d'autres termes, l'opération consistant, d'après un plan, à transformer une multitude de forces de travail individuelles en une seule et même force de travail sociale, n'est pas réductible à une "administration des choses ", à une opération de simple technologie sociale.

Il manque donc à Marx, en dépit de certaines de ses intuitions, une analyse du plan comme rapport social de production. La remarque vaut également pour le marxisme ultérieur. Trotski, par exemple, dont le mérite est d'apercevoir que, sur la base des contradictions d'une économie étatisée et planifiée, peut s'édifier une bureaucratie, refuse néanmoins d'envisager que cette couche sociale puisse intervenir dès le procès de production (il circonscrit son action à la sphère de la répartition). A-t-on besoin de dire que l'absence d'une caractérisation du plan comme facteur possible de classe a pesé lourd dans le destin des régimes se réclamant du marxisme? Il ne s'agit pas, néanmoins, pour Artous, de rendre Marx responsable de ce destin, mais seulement d'admettre que les erreurs commises dans les pays du "socialisme réel » sont venues combler des lacunes internes à la théorie de Marx, en jetant sur elles une lumière crue. $\mathrm{Au}$ final, on sort de la lecture de l'ouvrage d'Artous convaincu de l'immense chantier théorique ouvert à tous ceux pour qui la critique des rapports sociaux capitalistes continue de devoir se nourrir de la référence à Marx. Quel statut accorder aux présuppositions juridico-politiques contenues dans les rapports marchands et organisationnels? Quel rapport entre capitalisme, marché et organisation? Comment penser, dans une société socialiste, l'articulation entre les formes marchandes, organisées et associatives de production? On le voit, il n'est plus souhaitable de se contenter de simples ajouts à la théorie de Marx, ni même de corrections à la marge. C'est à une tâche de reconstruction du marxisme que les marxistes contemporains sont confrontés. En dégageant la possibilité d'un fétichisme de l'organisation, en établissant que le capitalisme se définit aussi par le développement de formes administratives spécifiques (et pas seulement par le marché), en soulignant que le socialisme ne saurait s'identifier à la forme organisée de production, nul doute que le travail d'Artous apporte une très utile contribution à cette entreprise. 


\section{AUTEURS}

\section{GUILLAUME COLLINET}

Enseignant en philosophie 